\title{
Modulation Transfer Microscopy - Versatile Tool for Ultrafast Nanoscopy
}

\author{
H. ORLikOWSKA* AND L. PiatKOWSKI \\ Institute of Physics, Faculty of Materials Engineering and Technical Physics, \\ Poznań University of Technology, Poznań, Poland \\ Doi: 10.12693/APhysPolA.139.288 *e-mail: hanna.orlikowska@put.poznan.pl
}

\begin{abstract}
Marrying the ability of single nanoparticle detection with the potential of tracking its rapid dynamics has provided unprecedented insights into micro- and nanoworld. Decoding the structural and dynamical phenomena at the single particle level, veiled in ensemble experiments, has a profound influence on our understanding of complex physical, chemical and biological processes. Yet, the majority of the techniques rely on the detection of fluorescence and therefore require efficient emissive species and are restricted to nanosecond-scale processes. Both limitations have prompted the advancement of a number of non-fluorescent detection methods, thereby pushing detection sensitivity, spatial and temporal resolution and imaging speed to the limits. In this mini-review, we discuss the recent progress in the time-resolved modulation transfer microscopy for label-free imaging and interrogating the ultrafast phenomena at the nanoscale. First, we cover the basic principles underlying the modulation transfer approach and put together an overview of its applications in different disciplines, specifying the exact contrast mechanism used. Next, we highlight the experimental challenges and limitations of the ultrafast modulation transfer microscopy methods. Finally, we address technical and experimental aspects yet to be realized in practice that potentially could further improve the discussed techniques.
\end{abstract}

topics: pump-probe microscopy, non-fluorescent detection, ultrafast spectroscopy, stimulated emission

\section{Introduction}

Even the most complex physical, chemical, and biological processes are determined by the fundamental, spatial, and temporal interaction trajectories at a single particle level. Ensemble experiments provide useful information about collective dynamics of a system, however only ultrafast techniques with single-particle sensitivity are able to track the photodynamics of single nanoparticles, unveiling their inherent transient intermediates. An ultimate dream of physicists, biologists, and chemists is to be able to peek into each particle and follow the movement of any atom in real time, i.e., to see every particle individually and pursue its femtosecond dynamics, to understand and be able to predict the function and real-time action of each particle within the greater process.

Three decades have passed since the first successful detection of individual molecules. The initial endeavours at cryogenic conditions [1], soon after the detection of individual fluorescent molecules at room temperature [2] and finally the time-resolved approach [3], rapidly paved the way towards routine investigation of molecular dynamics, chemical reactions, charge dynamics, structural and dynamical heterogeneities and much more. The discrete blinking and bleaching of single molecules has promptly developed as the essential trick behind modern super resolution microscopies [4]. Ultimately, with the advent of broadband lasers and advanced pulse shaping, the first femto-picosecond, intra- and interparticle dynamics of individual particles could be captured.

The recent merger of ultrafast spectroscopy with the detection on the nanoscale sparked a number of unique experiments on individual molecules, molecular complexes, and nanodevices, exploring processes such as molecular vibrations and motions [5, 6], vibronic spectral evolution [7], nanoscale plasmonic confinement $[8,9]$, coherent energy transfer [10], or photo-thermal decay in 2D materials [11]. Still, femtosecond processes on the nanoscale are notoriously difficult to access. The small interaction cross-sections of individual nanoparticles make it hard to rely on the conventional ultrafast approaches, such as transient absorption and nonlinear four-wave mixing. Consequently, single molecules and nanoparticles are almost exclusively detected through spontaneous emission (fluorescence or photoluminescence (PL)), which owing to the Stokes shift is background-free and has photon counting sensitivity, allowing for the detection of weakly fluorescent emitters. While exploited in super-resolution and 3D imaging, the use of fluorescence detection for many systems is hampered by a number of limitations: it is restricted to luminescent samples and sensitive to bleaching, and in the linear regime it is slow (with a nanosecond time response), hence reporting only on the population of the final emitting state, while missing out on femtosecond dynamics. 
While different chemical strategies have been developed to improve photostability of chromophores [12], huge effort has been made to develop imaging techniques based on contrasts other than fluorescence. Consequently, several alternative single emitter detection schemes have been explored in recent years, such as photo-thermal contrast [13], interferometric scattering microscopy [14], linear absorption [15, 16] or various enhanced Raman schemes [5, 17, 18], just to mention a few. Especially the recently presented time-resolved surface enhanced coherent anti-Stokes Raman spectroscopy (CARS) on a single molecule at room temperature is exciting and an important experimental achievement that places this technique head-tohead with the time-resolved, fluorescence-based single molecule detection $[5,18]$.

However, relying on plasmonic enhancement requires sculpting the environment in the vicinity of the molecule and to a large extent converting the system into a molecule-nanostructure hybrid, far from any resemblance to particles' natural state. These great experimental achievements truly pushed the boundaries for room-temperature single particle detection. Yet, their applicability has still shown intrinsic limitations, in temporal resolution, state selectivity or signal-to-noise ratio. Therefore, there is a continuous strive for new, universal experimental techniques that with high specificity would be capable of precise, non-invasive, fluorescence-free, ultrafast detection of single entities, beyond the slow and photo-fragile fluorescence detection.

One of the experimental approaches, which aspires to take the lead in ultrafast nanoscopy is the microscopy version of the pump-probe spectroscopy method, routinely used to access ultrafast dynamics in bulk systems. The pump-probe methodology is based on two laser pulse interactions with the sample and transferring of modulation from the pump beam to the probe beam. The method has many variants and may involve various interactions between the laser pulses and the sample. It is often referred to in the literature as transient absorption microscopy, analogously to bulk transient absorption spectroscopy. Transient absorption, however, indicates that the contrast results directly from the change of the absorbance of the sample, which is the case for processes such as ground state depletion or excited state absorption. But one of the possible interactions - stimulated emission — depends on the population of the excited state and does not involve absorption of a photon. Hence, modulation transfer microscopy seems to be the more appropriate term to encompass all possible interactions and consequently contrast sources.

In this mini-review, we first discuss the concept of modulation transfer microscopy with particular focus on the time-resolved approach to the examination of nanoscopic systems. We provide a concise overview of modulation transfer microscopy applications across a number of research fields. Then, we describe our contribution to the field based on our recent development of the femtosecond, stimulated emission microscopy to study charge dynamics in quantum dots. Finally, we address the challenges, limitations and perspectives of modulation transfer microscopy in the framework of ultrafast nanoscopy.

\section{Basics of modulation transfer microscopy}

In a pump-probe imaging technique, referred to in this review as modulation transfer microscopy, modulation of one of the incident beams imposes modulation on the other beam upon nonlinear interaction with the sample. In a typical experiment, a pair of short laser (femtosecond or picosecond) pulses is used. The first one (the pump) promotes a sample to its electronic excited state, whereas the second pulse (the probe) interrogates its transient states. The two pulse trains are temporally synchronized and the pump-probe interpulse delay time is precisely controlled. The beams are spatially combined before being focused on a sample with a high numerical aperture objective. Prior to the sample, the pump beam is modulated with a high frequency, typically at the level of several megahertz, selected to overcome the noise spectrum of laser fluctuations, using an external shutter (acousto- or electrooptic modulator). After interaction with the sample, the pump light is rejected and the probe beam, to which the modulation has been transferred, is detected using a photodiode either in transmission or in epimode, depending on the transparency of the sample. The readout of the photodetector is then demodulated using a lock-in amplifier, referenced to the pump beam modulation frequency, to extract the depth of modulation. A general simplified excitation and detection scheme of modulation transfer microscopy is depicted in Fig. 1a.

When the pump-induced change in the probe intensity is due to the involvement of electronically excited states of the analyte, three major types of processes are considered: excited state absorption (ESA), stimulated emission (SE), and ground state depletion (GSD) (see Fig. 1b).

Once the excited states of the sample are populated upon the absorption of pump photons, three scenarios are possible: (i) the photoexcited system absorbs the photon energy of the subsequent probe pulse and undergoes the ESA, which results in the reduction of the probe beam transmission, (ii) the probe pulse stimulates the sample back to the ground state with a creation of new coherent photons, leading to an increase of the probe intensity (SE), and/or (iii) the probe beam induces the transitions from the ground to excited states. Because of the transient depletion of the ground state population, the probe experiences a decreased attenuation, hence a relative gain of its intensity is observed (GSD) (Fig. 1c). 


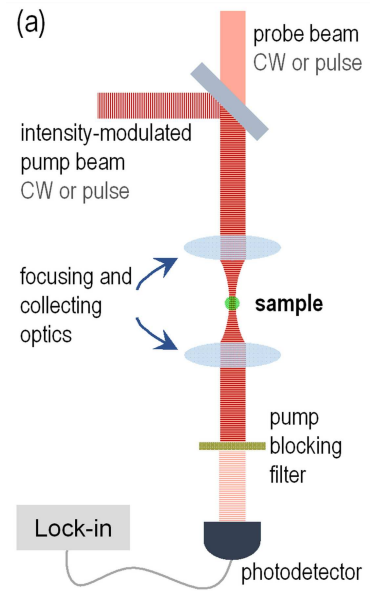

(b) Relative energy of the pump $(\uparrow)$ and probe $(\uparrow)$ beams

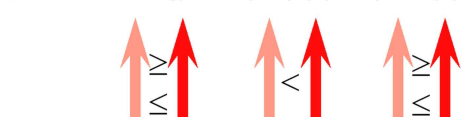

(c)
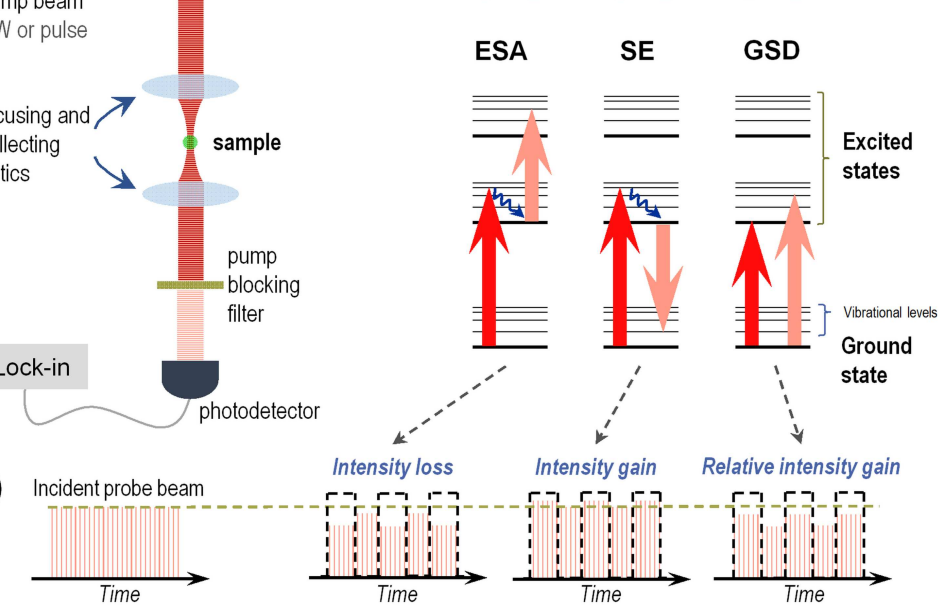

Relative intensity gain

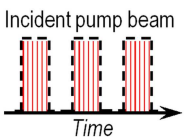

Fig. 1. Basic concepts of modulation transfer microscopy. (a) General schematic of experimental setup. The principle of operation is described in the main text. (b) Energy diagram of the three main possible processes contributing to the modulation transfer signal: ESA, SE, and GSD of an exemplary analyte. (c) Schematic of modulation transfer - temporal characteristic of pump and probe pulse trains before and after interaction with the sample. With the pump beam on, the probe experiences either a loss or a gain in its intensity. For reference, the modulation of the pump beam is shown as dashed square wave.

Depending on the probe energy, with respect to the pump beam, the acquired contrast can simply arise from a single process or may result from the interplay between ESA, SE, and GSD (see Fig. 1b). ESA can occur in all cases: when the probe energy is higher than the pump energy, when it is lower, and equal to the pump energy, depending on the transition energy to higher excited states. SE only occurs when the probe energy is lower than energy of the pump and is resonant with the emission spectrum of the analyte (or, in the case of the nonfluorescent system, simply red-shifted with respect to absorption). GSD can occur both when the probe energy is greater than the pump energy and when the energies are equal. While SE is relatively easy to distinguish from GSD, the separation of ESA is not trivial. Nevertheless, the relative contribution of contrast components depends not only on the experimental conditions, but also on the exact nature of the sample, which is demonstrated in our recent article [19].

Regardless of which processes contribute to the acquired contrast, under the unsaturated conditions, the depth of modulation is proportional to the product of the pump intensity, the probe intensity and the analyte concentration. The overall quadratic intensity dependence provides intrinsic optical sectioning, while the linear dependence on analyte concentration permits straightforward quantification [20]. Pump-induced changes in the probe beam intensity (the depth of modulation) can be measured as a function of the time delay between the pump and probe pulses and thus the excited state relaxation dynamics can be monitored. The acquired time traces exhibit different temporal signatures characteristic of the analyte, ensuring high detection specificity and can serve to determine the origin of the contrast.

Note that the contributions to a modulation transfer signal are not limited to the three abovementioned processes. Other nonlinear optical interactions, accessible with pump-probe microscopy, which may serve as an imaging contrast include: stimulated Raman scattering (SRS), two-photon absorption (TPA), photothermal effect (PTE), and cross-phase modulation (XPM). However, in this review we focus solely on the processes that involve real intermediate states. For a comprehensive overview of optical nonlinearities, which have proved to be useful in acquiring contrast in microscopy, see [21, 22].

It should be noted that the ESA, SE, and GSD processes are not unique to organic chromophores, for which an exemplary energy level diagram is depicted in Fig. 1b. Analogous optical interactions can occur in inorganic conducting $[23,24]$ and semiconducting systems [19], as demonstrated in the following sections. 


\section{Overview of modulation transfer microscopy applications}

The first article devoted to the use of modulation transfer microscopy was published by Dong et al. in the 1990s [25]. The authors took advantage of stimulated emission for lifetime-resolved imaging of human erythrocytes and mouse fibroblast cells labeled with exogenous fluorophores. After over a decade, Warren's group, utilizing high-frequency modulation scheme, advanced the technique towards imaging of non-fluorescent endogenous biopigments (melanin [26, 27] and hemoglobin [28]) in a labelfree manner. In the follow-up study, they demonstrated the feasibility of this approach to differentiate between eumelanin and pheomelanin [29]. Vastly different transient response from the two chemically distinct forms of melanin was used for the skin cancer diagnosis in the early stages, both ex vivo and in vivo [29-31]. In addition, Wilson et al. by visualizing biopigments distribution demonstrated the potential of modulation transfer microscopy for use as a noninvasive optical biopsy technique to support the clinical and surgical management of ocular melanocytic lesions [32].

Intrinsic optical properties of endogenous hemoglobin were utilized for label-free imaging of microvasculature structure [28, 33] and for measuring blood flow velocity in live model animals [34]. In addition, distinct excited state dynamics of oxyhemoglobin and deoxyhemoglobin made it possible to distinguish between arterioles and venules [35] and to quantify the oxygen saturation on a single red blood cell level in real-time [36]. Under conditions of long-term high blood sugar, hemoglobin chemically links to glucose, forming a compound commonly used to diagnose diabetes. Different time-resolved decay signature of glycated hemoglobin from unmodified hemoglobin was exploited by Dong et al. to quantify glycated hemoglobin fraction inside a single red blood cell [37]. Other heme species studied with modulation transfer approach include free heme (hemin) [38], respiratory chain pigment [39, 40] and hemoglobin degradation product hemosiderin [41].

Besides applications to endogenous nonfluorescent biopigments, modulation transfer microscopy has also been adapted to investigate exogenous contrasts such as drugs [33] and nanomaterials in living cells, tissues and animals [42]. This modality has provided informative images to monitor the location, dynamics and interactions of several types of nanostructures, including gold nanorods [43], nanodiamonds [44], graphene and graphene oxide layers [45] and single-walled carbon nanotubes [23] inserted into biological tissues.

Modulation transfer microscopy has also been exploited for nondestructive investigation of historical artworks by generating distinctive pump-probe contrast from a range of inorganic and organic paint pigments [46-50].
In materials science, modulation transfer approach has been extensively utilized for imaging and characterization of materials ranging from semiconducting thin films, through molecular and nanostructured assemblies, to nanoscale interfaces and single nanostructures and molecules [16, 51-53].

By capturing the distinct exciton dynamical signatures arising from different domains of the polymer blend, Wong et al. unraveled the relationship between ultrafast photophysics and film morphology, necessary to optimize the performance of organic photovoltaic devices [54]. Jung et al., in turn, exploited the phase of the modulation transfer signal as a contrast to visualize and discriminate between metallic and semiconducting individual single-walled carbon nanotubes [24]. Further, Lo et al. studied the carrier trapping dynamics of single CdTe nanowires, demonstrating that the excited state behaviors vary substantially from wire to wire and are strongly dependent on trap-filling [55]. Moreover, they observed for the first time a coherently excited phonon modes in single semiconductor nanostructures [55].

Ultrafast pump-probe microscopy proved to be useful not only to reveal variation in kinetic behavior across an ensemble of nanoobjects, but also to unveil that the excited state dynamics can vary within a single nanostructure [56]. Mehl et al. investigated the electron-hole recombination dynamics at different locations within a single tapered $\mathrm{ZnO}$ nanorods [57, 58]. Time-resolved images exposed two different decay pathways, the recombination dominated by trapping at points in the interior and dramatically faster recombination through the electron-hole plasma mechanism at the ends of the rod. On the other hand, Grumstrup et al. studied the strain-induced effects on the electronic dynamics across an individual Si nanowires [59]. They demonstrated that in the bent segments, the semiconductor nanostructure exhibit significantly faster electron-hole recombination than in the straight regions. Since the interaction with a substrate can affect the properties of nanomaterials, Gao and co-workers compared the modulation transfer signatures of the suspended and substrate-supported sections of the individual chirality-assigned singlewalled carbon nanotube, disclosing the role of dielectric screening effects [60]. The detection of the modulation transfer signal with simultaneous fluorescence recording led Chong and coworkers to achieve the ultimate sensitivity and to detect an absorption signal from a single Atto647N molecule at room temperature [16].

A summary of selected applications in various research areas along with the dominant contrast mechanism is presented in Fig. 2. They were grouped according to whether modulation transfer microscopy was used to examine samples at the level of a single particle or its assemblies, and whether it focused on imaging or on revealing the dynamics of photoinduced processes. 


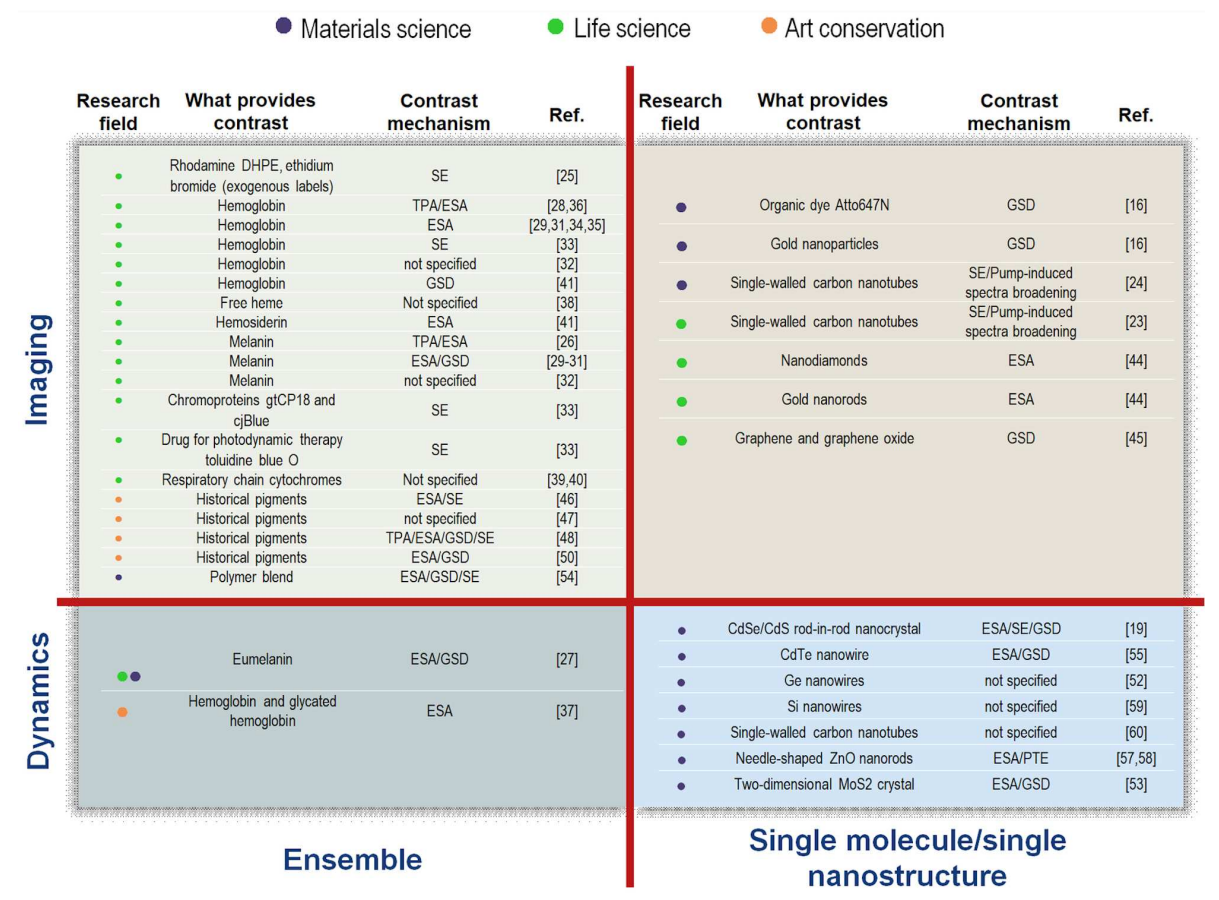

Fig. 2. A summary of selected applications of modulation transfer microscopy in various research areas along with the dominant contrast mechanism. For more details, see the original articles.

The aim of this article is to highlight the versatility of the modulation transfer technique. Therefore, for more field-specific examples we refer the reader to the following articles, e.g., focused on the study of single nanostructures [56], devoted to the applications to solar energy harvesting systems [51] or recent technological advancements-oriented [61].

\section{State of the art}

We have recently built up on the concept of ultrafast modulation transfer microscopy and, for the first time, presented direct stimulated emission detection and imaging of individual semiconducting nanocrystals (NCs) while at the same time tracing their excited state dynamics with femtosecond temporal resolution at ambient conditions [19]. In the experiment, the spontaneous emission (photoluminescence, PL) is detected simultaneously with the SE, generating two independent, complementary contrast images. We applied the method to colloidal CdSe/CdS rod-in-rod NCs. Beyond application as imaging labels [62, 63], colloidal NCs have in recent years gained a particular interest. Today they are successfully applied in lasers [64-66], displays [67], light emitting diodes [68-70], photovoltaics [71-73], light detection [74] and bioimaging $[75,76]$. This is thanks to the growing ability to engineer NCs not only with different composition, geometry and emission wavelength, but with pre-designed quantum properties, that is the energy states manifold [77].

For any potential application, competition between radiative and non-radiative charge relaxation and transfer channels plays a crucial role. Therefore, understanding the dynamics and interplay between various charge relaxation pathways, such as charge transfer, injection and extraction, charge delocalization and excited state relaxation, with high temporal resolution and nanoscopic sensitivity is of paramount importance. In our article [19], we demonstrated that the femtosecond stimulated emission experiment on single-NCs delivers a wide range of information regarding excited state relaxation dynamics of individual charges, dynamical heterogeneity of NCs and the relative contributions of SE, GSD, and ESA processes, all with single-NC sensitivity.

Details on the technical issues can be found in the original publication and its supplementary information. However, in brief, in the experiment we used a broadband $(\approx 200 \mathrm{~nm}$ ) laser that, in combination with the dispersion control stage (based on dispersion mirrors and liquid-crystal based pulse shaper), delivers nearly transform-limited pulses at the sample plane (see Fig. 3 and the original work). The pump beam was propagated through an acoustooptic modulator (AOM) operating at high $(\mathrm{MHz})$ frequency. The two pulse trains were then recombined, coupled into an inverted microscope and focused onto the sample with a high NA microscope objective. Spontaneous emission from the sample was collected in epi-configuration and detected spectrally using an EM-CCD camera equipped with a spectrograph, or using an avalanche photodiode. The stimulation beam was detected in transmission and the signal was demodulated using a lock-in amplifier. 

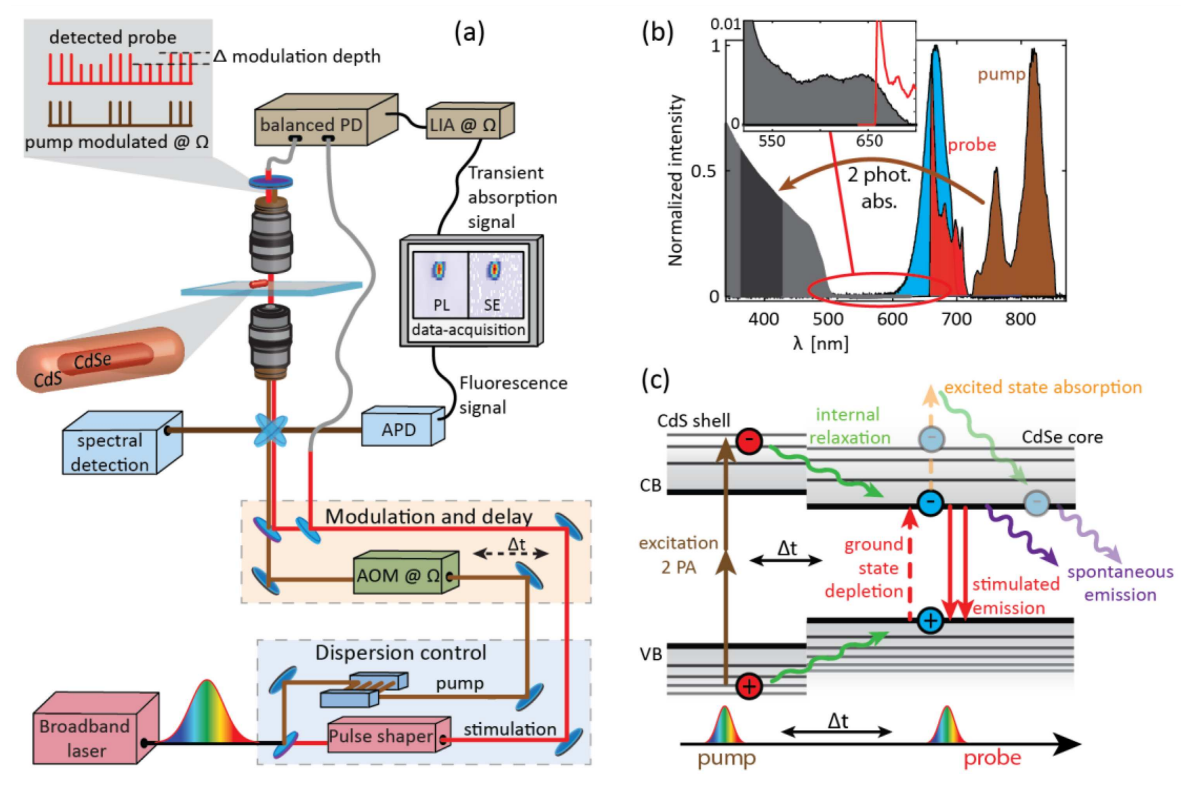

Fig. 3. (a) Schematic of the experimental setup. (b) Spectral characteristics of the laser pulses and CdSe/CdS NCs. Brown and red shaded areas represent pump and probe parts of the broadband laser spectrum, respectively. Grey and blue shaded areas represent absorption and emission spectra of the NCs, respectively. The black area indicates the spectral range of the two-photon absorption. (c) Energy level sketch of a core/shell CdSe/CdS NC and a schematic of possible interactions with the pump and probe pulses [19].

In the experiment, the two-photon absorption in the shell of the nanocrystal is followed by the internal relaxation of the excited charges. A timedelayed probe pulse, resonant with the core transition, probes the excited state of the NC. Each excitation event leads to a net increase of the probe beam intensity due to stimulated emission and/or ground state depletion process. Figure 4a shows side-by-side images of two individual NC obtained with PL contrast (top row) and with modulated signal (bottom) for three different delay times between the pump and probe pulses. Figure $3 \mathrm{~b}$ shows time traces acquired from a single NC. The correlation between the depletion of the PL signal and an increase in the modulated signal clearly indicates that they probe the excited state population. Positive sign of the modulated signal confirms a net increase in the probe beam intensity, confirming that the two main contributions come from GSD and SE. For details on the interpretation of the data please see the original work [19].

The so designed experiment allowed us to image individual nanocrystals through two complementary contrasts and then gain direct access to the dynamics of individual charge carriers in these structures. The experiments are unique in many ways, however special attention should be paid to three key aspects: (i) simultaneous detection of two, complementary contrasts, (ii) determination of the photon budget (absolute numbers) from two different detection stages and (iii) probe pulse duration dependence experiment gave quantitative insights into importance of the ESA process. To elaborate on these points:
- Combined with simultaneous detection of PL, the experiment allowed us to provide a complete picture of the excited charges, which are either stimulated down, or promoted to higher excited states, or they recombine spontaneously. In a conventional steady state experiment, GSD and SE processes lead to a net increase of the probe beam intensity, hence are indistinguishable. Acquiring the timeresolved traces allowed us to differentiate between the GSD and SE contributions. This was aided by the fact that the two excited charges, electrons and holes, exhibit different relaxation times [62, 78, 89]. In a nutshell, we concluded that in the studied NCs excited holes localize at the core band edge within $200 \mathrm{fs}$, while the excited electrons relax to the core band edge on a time scale of $550 \mathrm{fs}$. Our experiment also allowed us to probe the intrinsic and/or locally induced dynamical heterogeneity among individual NCs. Comparing the slow charge (electron) dynamics from multiple measurements we found that the charge relaxation time differs nearly a factor of two between individual NCs. The disparity in charge relaxation dynamics allowed us to determine the relative amplitudes of SE and GSD, showing that on average they comprise $<20 \%$ and $>80 \%$ of the total induced ground/excite state population difference, respectively.

- The single-emitter sensitivity of our experiment allowed us to compare the number of photons lost in $\mathrm{PL}$ and gained through SE in absolute terms, which is difficult to achieve for ensembles [66]. Given that the stimulated emission signal corresponds to the missing excited state population, the number of photons detected in SE should be equal to the 


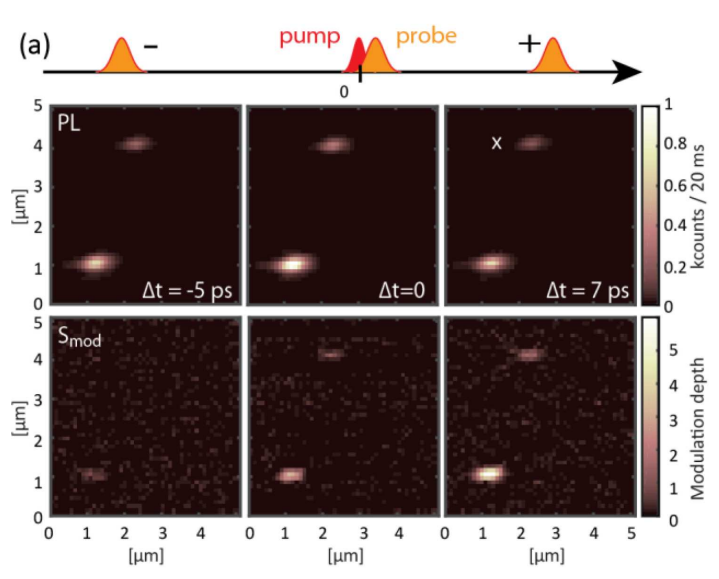

(b)

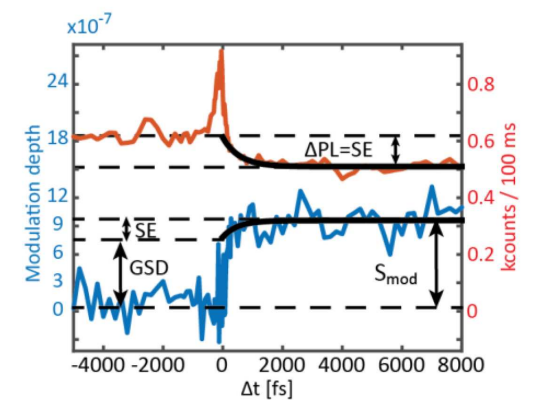

Fig. 4. (a) A series of images acquired by detecting PL (top row) and $S_{\text {mod }}$ (bottom row) signal, for different excitation and stimulation interpulse delays $\Delta t$ (negative $-5 \mathrm{ps}, 0 \mathrm{ps}$ and positive $+7 \mathrm{ps}$ ). (b) Simultaneously detected $S_{\bmod }$ (blue) and PL (red) time traces for a CdSe/CdS rod-in-rod NC [19].

number of photons missing in the PL, that is PL depletion. Using a simple calculation that takes into account detection efficiencies of the PL and modulated signal, we determined an effective number of photons depleted from PL, photons/second and an effective number of photons gained in the stimulation beam photons/second per individual NC. The two values are in good agreement, given the fact that the detection occurs in two independent channels and using different detector types (analogue vs photon counting).

- To account for all the possible interactions between the probe pulse and the excited charges, we performed stimulation pulse duration dependence experiments, which allowed us to elucidate the presence of ESA. Lengthening of probe pulses also allows decreasing the impact of ESA by allowing the system to develop in the excited state and possibly to stimulate the system in a secondary interaction after it underwent ESA [19]. This is analogous to STED experiment, where stretching the stimulating pulse allows for longer interaction time between the pulse and the sample, allowing the system to evolve in the vibrationally hot ground state, decreasing the probability of follow up excitation back to the excited state and increasing the efficiency of STED process [80]. We found that stretching the stimulation pulse from 50 fs to about 2 ps increased the SE efficiency by $40 \%-50 \%$. This means that a significant portion of the excited charges undergo ESA and relax back to the core band edge states. A simple four-level model qualitatively reproduced our experimental data.

In summary, in the discussed work, we reported the direct detection of stimulated emission from individual NCs at ambient conditions. From a single modulation transfer experiment, we could determine the exact contributions of GSD and SE to the measured signals and relative importance of the SE and ESA processes, all with femtosecond time resolution and single-emitter sensitivity.

\section{Challenges in ultrafast modulation transfer microscopy}

Transferring the modulation transfer (pumpprobe) scheme from ensemble studies to microscopy may seem trivial, however specific technical aspects need to be considered. An absolute prerequisite to ultrafast microscopy is the ability to control short laser pulses in the focus of a confocal microscope. In this regard, three key requirements need to be fulfilled:

- Laser pulses undergo significant phase/time distortions when propagated through optical elements, in particular high numerical aperture microscope objectives, comprised of a number of thick glass components [81]. One thus needs to be able to compensate for and then control the dispersion of the laser pulses. Different strategies were employed in recent years, mainly involving the use of second harmonic particles (since second-harmonic generation, being nonlinear coherent process, is extremely sensitive to pulse duration) such as barium titanate $\left(\mathrm{BaTiO}_{3}\right)$, iron iodate $\left(\mathrm{Fe}\left(\mathrm{IO}_{3}\right)_{3}\right)$ or gold $(\mathrm{Au})$ in combination with experimental schemes such as frequency-resolved optical grating (FROG) [82] or spectral phase interferometry for direct electric-field reconstruction of ultrashort optical pulses (SPIDER) [83]. In our works, we used yet another approach called multiphoton intra-pulse interference phase scan (MIIPS) $[19,84,85]$. In combination with a pulse shaper and sub-diffraction limit second harmonic nanoparticles, it allowed to compress laser pulses in the focal plane of a high NA objective to a Fourier limit. This approach also allowed us to characterize the focal spot in terms of uniformity and chromatic aberration - issues described below.

- In a typical microscopy experiment, the achievable spatial resolution is limited by the diffraction limit, which is much larger than the size of a typical nano-object under study. Hence, the focal spot of a laser beam needs to be highly uniform in terms of spatiotemporal characteristic. Spatiotemporal distortions may become particularly apparent when pulse shapers are used [86]. However, it is relatively easy to characterize the focal spot using second harmonic nanoparticles — in such a case, the object to 
be studied becomes a probe for the spatiotemporal uniformity of the focal spot. The nanoparticle is then scanned across the focal spot while monitoring the second harmonic emission from the particle, revealing any spectral and temporal heterogeneity within the focal spot. Such characterization and control is required so that when addressing multiple, individual nano-objects, the measured heterogeneity originates solely from their inherit, structural and dynamical properties of the subject and not from the varying interaction between the nanoobject and the laser pulse.

- Last but not least, the pump-probe approach typically involves laser pulses in the visible range with the same or similar wavelengths. However, sometimes it is beneficial to use laser pulses that are spectrally far from each other, for instance when one of the pulses is involved in a single photon interaction and the other is involved in a two-photon interaction. In such a case, chromatic aberration affects the spatial overlap of the pump and probe pulses in the focal spot, significantly decreasing the simultaneous interactions of the nano-object with these pulses. Typical microscope objectives have chromatic aberration correction in the visible range, typically $500-650 \mathrm{~nm}$. Using wavelengths outside this range one should characterize the optical system in terms of chromatic aberration. This can be done by placing second harmonic nanoparticles (for instance, the aforementioned $\mathrm{BaTiO}_{3}$ nanoparticles); scanning the nanoparticle in the direction of laser beams propagation and verifying whether the observed second harmonic signals from the two laser pulses are generated most efficiently for exactly the same nanoparticle position. Even if chromatic aberration cannot be corrected and/or avoided, it is crucial for interpretation of the experimental data to know the strength of the interaction between the object and the pump and probe pulses.

\section{Limitations}

The discussed approach, just like any other experimental technique, is not free from shortcomings:

- The first and foremost is that the acquired signals (irrespective of the origin — GSD/SE/ESA) are weak (much weaker than the intensity of the probe beam) and superimposed on high intensity background. Consequently, phase sensitive lock-in detection needs to be applied to extract the minuscule change of the probe beam intensity. The expected modulation depth for a single chromophore is $\approx 10^{-7}$. Such a small signal can be detected in the experiment with optimized conditions, both for GSD [87] and SE [19]. However, with an ultimate detection limit of about $10^{-7.5}-10^{-8}$ given by the shot noise, any imperfection in setting up the experiment may prevent us from the detection of individual emitters or even small clusters.

- The acquired signal is often hard to interpret in terms of the origin of the contrast. GSD and
SE can be reasonably well distinguished by simply spectrally tuning the excitation and probe beams with respect to each other. With particular settings, when the pump beam has lower energy than the probe beam [87], or the other way around, when the probe beam has lower energy and is redshifted outside the absorption band of the studied object, the contrast originates in principle solely from GSD and SE, respectively. However, separating ESA from GSD or SE is much more difficult, especially that most of the time strengths and spectral positions of the excited states transitions are unknown. In such a situation, ensemble transient absorption experiments can be very helpful as shown by Hotta et al. [63]. In the case of our studies, the separation of GSD/SE/ESA contributions to the measured signal was aided by the fact that the probed charges (electrons and holes) have different relaxation times - the faster of the two leads to a nearly instantaneous appearance of the GSD, while the slower one leads to a gradual rise of the SE signal.

- Modulation transfer microscopy depends on two distinct frequencies (excitation and stimulation) making it intrinsically confocal. The interaction between the sample and the two fields in combination with the inter-pulse time dependence makes the method quite sensitive to different species within a dense ensemble [19]. However, it should be noted that the selectivity and specificity are not as good as for various Raman schemes.

- Finally, the time-resolved modulation transfer microscopy requires rather complex experimental setup - ultrafast, tunable laser; dispersion control; microscopy stage; sensitive detection stages and meticulous alignment, which altogether make the approach far from being routine, every-day experiment.

\section{Perspectives}

Modulation transfer microscopy has been around for three decades now and has proven to be a valuable tool. Yet, not all the capabilities have been explored, especially in the ultrafast nanoscopy and single nano-object field. It seems thus appropriate to briefly summarize, based on our experience, what further modifications and experiments could be implemented in order to explore the applicability and limits of this techniques:

- The majority of modulation transfer microscopy experiments involve single photon interactions between the sample and the pump and probe beams. For samples absorbing towards UV (especially those of biological character) two-photon excitation and/or probing is beneficial: first to avoid photodamage and second to be able to use ultrafast lasers (typically Ti:sapphire) delivering short pulses in the near IR region. Due to low two-photon interaction cross-sections this approach is rarely used. In our experiment, we used broadband laser pulses in the near IR spectral region. The higher spectral 
range was used for two-photon excitation of a quantum dot, while the lower spectral region was used for single-photon probing. Other single/two-photon pumping/probing configurations could be explored.

- When small signals are being detected, any variation in the pump and probe beams intensities should be avoided and/or compensated for. The effect of intensity fluctuations of the probe beam on the measured signal can be diminished using a balanced detection. The probe beam transmitted through the sample and a reference probe beam (part of the probe beam split before interacting with the sample) are fed to a balanced detector, which subtracts a slowly varying component from the probe beam signal, increasing the overall signal to noise ratio of the detected signal. The advantage of this approach was demonstrated in an experiment where single molecules were detected through optical absorption [15, 88].

- Since SE is a coherent process, the experimental setup could be expanded to accommodate heterodyne detection, in which the weak modulated signal can be amplified significantly by mixing it with the so-called local oscillator of high intensity. This could be particularly useful for applications, where intense probe beam is undesired. The weak probe beam carrying a minuscule signal can be amplified many orders of magnitude and brought into the detection range [89].

- As mentioned earlier, the efficiency of stimulated emission depletion (STED) process has been shown to depend significantly on the temporal length of the stimulation pulses. We inversed this idea to the excited state and showed that lengthening of the stimulating pulse can also increase the efficiency of SE by allowing the system to evolve in the excited state, that is to undergo ESA. Hence determining how all the signals scale with the length of the stimulation pulse, one can estimate the relative importance of the ESA process with respect to GSD and SE, in other words one can quantitatively map all the interactions involved.

- Characterization of an excited system is often limited to the exploration of lowest excited states. However, studying of higher excited states, their relaxation pathways and rates is important in the framework of applications in photovoltaics and material sciences. Being able to determine the relaxation rates of selected excited states of quantum dots for instance would be of great value [90]. Such a selective probing of the excited state manifold and state-to-state dynamics would require spectral scanning of the probe beam, which could be achieved by using femtosecond, near infrared laser pulses and probing via two-photon interaction.

- Since the stimulated emission is a coherent process, it opens up possibilities to study coherent effect, such as excited state coherent energy transfer in light harvesting antenna complexes [10, 67]. So far, such experiments relied on fluorescence detection. Utilizing a coherent contrast would allow for direct probing of coherent processes in the excited state and provide deeper insights into quantum effects in biological and biomimetic systems.

- Spatial imaging with high temporal and spatial resolution of nanoscopic samples would be another application, where stimulated emission microscopy could prove its value. Ultrafast pump-probe approach based on thermomodulation microscopy has recently been used to study electron dynamics in thin metal films [91]. Various approaches for spatial imaging with femtosecond resolution were developed in the Huang group [51, 92]. These studies were predominantly based on transient absorption or transient reflectance contrast and were used to address dynamics in thin film samples.

\section{Conclusions}

Inherent, unique and often heterogeneous properties of matter are strongly influenced by the immediate environment at the nanoscale. As a consequence, a bottom-up approach to many technological problems is increasingly used and consists of systematic, comprehensive, fundamental research on simple, often model systems, which then allow for a better understanding of macroscopic behavior of the systems and for predicting their evolution in time. However, the main and recurrent obstacle to this approach is the limited availability and applicability of different experimental methods to characterize various systems at the nanoscale. Years ago, Professor Sydney Brenner, winner of the 2002 Nobel Prize in Medicine, said: "Progress in science depends on new techniques, new discoveries, and new ideas, probably in that order".

Undeniably, scientific progress often depends on the development or discovery of new experimental techniques. An example of this is the development of fluorescence microscopy. Inconspicuous, or at least very fundamental in its nature experiment, allowing detection of individual molecules by recording their fluorescence [2], very quickly initiated a technological revolution that led in 2014 to awarding the Nobel Prize in Chemistry to Stefan Hell, William Moerner and Eric Betzig for their contribution to the development of (super-resolution) fluorescence microscopy. It has by now variants and is used nearly routinely in almost all branches of exact sciences.

Various time-resolved microscopy approaches have been developed in recent years breaking yet again temporal, spatial and sensitivity limits. Scouting for new sources of contrast that would provide deeper insights into the structure and dynamics of matter on the nanoscale and that would compete with fluorescence detection continues. In the years to come we will surely observe increasing efforts in mastering, optimization and development of new techniques, pushing boundaries of how small, how fast, how precisely and for how long we can study the nanoworld. 


\section{Acknowledgments}

The authors acknowledge financial support from the EMBO Installation Grant 2019 and from the Ministry of Science and Higher Education of Poland. LP acknowledges the financial support from the First TEAM grant No. POIR.04.04.0000-5D32/18-00, provided by the Foundation for Polish Science (FNP). This work was financed from the budget funds allocated for science in the years 2019-2023 as a research project under the "Diamond Grant" program (Decision No. 0042/DIA/2019/48). The work was also financed by the Ministry of Science and Higher Education in Poland in 2020 under Project No. 0512/SBAD/6209.

\section{References}

[1] W.E. Moerner, L. Kador, Phys. Rev. Lett. 62, 2535 (1989).

[2] M. Orrit, J. Bernard, Phys. Rev. Lett. 65, 2716 (1990).

[3] E. Betzig, R.J. Chichester, Science 262, 1422 (1993).

[4] X.S. Xie, R.C. Dunn, Science 265, 361 (1994).

[5] S. Yampolsky, D.A. Fishman, S. Dey, E. Hulkko, M. Banik, E.O. Potma, V.A. Apkarian, Nat. Photon. 8, 650 (2014).

[6] D. Brinks, F.D. Stefani, F. Kulzer, R. Hildner, T.H. Taminiau, Y. Avlasevich, K. Müllen, N.F. Van Hulst, Nature 465, 905 (2010).

[7] M. Liebel, C. Toninelli, N.F. Van Hulst, Nat. Photon. 12, 45 (2018).

[8] M. Aeschlimann, T. Brixner, A. Fischer et al., Science 333, 1723 (2011).

[9] V. Kravtsov, R. Ulbricht, J.M. Atkin, M.B. Raschke, Nat. Nanotechnol. 11, 459 (2016).

[10] R. Hildner, D. Brinks, J.B. Nieder, R.J. Cogdell, N.F. Van Hulst, Science 340, 1448 (2013).

[11] K.J. Tielrooij, L. Piatkowski, M. Massicotte et al., Nat. Nanotechnol. 10, 437 (2015).

[12] J.H.M. Van Der Velde, J. Oelerich, J. Huang et al., Nat. Commun. 7, 1 (2016).

[13] A. Gaiduk, M. Yorulmaz, P.V. Ruijgrok, M. Orrit, Science 330, 353 (2010).

[14] G. De Wit, J.S.H. Danial, P. Kukura, M.I. Wallace, Proc. Natl. Acad. Sci. USA 112, 12299 (2015).

[15] P. Kukura, M. Celebrano, A. Renn, V. Sandoghdar, J. Phys. Chem. Lett. 1, 3323 (2010).
[16] S. Chong, W. Min, X.S. Xie, J. Phys. Chem. Lett. 1, 3316 (2010).

[17] A.B. Zrimsek, N. Chiang, M. Mattei, S. Zaleski, M.O. McAnally, C.T. Chapman, A.I. Henry, G.C. Schatz, R.P. Van Duyne, Chem. Rev. 117, 7583 (2017).

[18] L. Piatkowski, J.T. Hugall, N.F. Van Hulst, Nat. Photon. 8, 589 (2014).

[19] L. Piatkowski, N. Accanto, G. Calbris, S. Christodoulou, I. Moreels, N.F. Van Hulst, Science 366, 1240 (2019).

[20] W. Min, C.W. Freudiger, S. Lu, X.S. Xie, Annu. Rev. Phys. Chem. 62, 507 (2011).

[21] M.C. Fischer, J.W. Wilson, F.E. Robles, W.S. Warren, Rev. Sci. Instrum. 87, 031101 (2016).

[22] W. Min, C.W. Freudiger, S. Lu, X.S. Xie, Annu. Rev. Phys. Chem. 62, 507 (2011).

[23] L. Tong, Y. Liu, B.D. Dolash, Y. Jung, M.N. Slipchenko, D.E. Bergstrom, J.X. Cheng, Nat. Nanotechnol. 7, 56 (2012).

[24] Y. Jung, M.N. Slipchenko, C.H. Liu, A.E. Ribbe, Z. Zhong, C. Yang, J.X. Cheng, Phys. Rev. Lett. 105, 217401 (2010).

[25] C.Y. Dong, P.T. So, T. French, E. Gratton, Biophys. J. 69, 2234 (1995).

[26] D. Fu, T. Ye, T.E. Matthews, G. Yurtsever, W.S. Warren, J. Biomed. Opt. 12, 054004 (2007).

[27] K.Y. Ju, M.C. Fischer, W.S. Warren, ACS Nano 12, 12050 (2018).

[28] D. Fu, T. Ye, T.E. Matthews, B.J. Chen, G. Yurtserver, W.S. Warren, Opt. Lett. 32, 2641 (2007).

[29] T.E. Matthews, J.W. Wilson, S. Degan, M.J. Simpson, J.Y. Jin, J.Y. Zhang, W.S. Warren, Biomed. Opt. Expr. 2, 1576 (2011).

[30] T.E. Matthews, I.R. Piletic, M.A. Selim, M.J. Simpson, W.S. Warren, Sci. Transl. Med. 3, 71 ra15 (2011).

[31] J.W. Wilson, S. Degan, M.A. Selim, J.Y. Zhang, W.S. Warren, Proc. SPIE 8226, 822602 (2012).

[32] J.W. Wilson, L. Vajzovic, F.E. Robles, T.J. Cummings, P. Mruthyunjaya, W.S. Warren, Invest. Ophthalmol. Vis. Sci. 54, 6867 (2013).

[33] W. Min, S. Lu, S. Chong, R. Roy, G.R. Holtom, X. Sunney Xie, Nature 461, 1105 (2009).

[34] T. Chen, Y. Huang, Anal. Chem. 89, 10120 (2017). 
[35] D. Fu, T.E. Matthews, T. Ye, I.R. Piletic, W.S. Warren, J. Biomed. Opt. 13, 040503 (2008).

[36] A.T. Francis, K. Berry, E.C. Thomas, A.H. Hill, D. Fu, J. Phys. Chem. Lett. 10, 3312 (2019).

[37] P.T. Dong, H. Lin, K.C. Huang, J.X. Cheng, Sci. Adv. 5, eaav0561 (2019).

[38] A.J. Chen, X. Yuan, J. Li, P. Dong, I. Hamza, J.X. Cheng, Anal. Chem. 90, 3395 (2018).

[39] S.R. Domingue, R.A. Bartels, A.J. Chicco, J.W. Wilson, Biomed. Opt. Express 8, 2807 (2017).

[40] J.W. Wilson, S.R. Domingue, R.A. Bartels, E. Wang, in: Ultrafast Nonlinear Imaging Spectrosc. V, Ed. Z. Liu, SPIE, 2017, p. 26.

[41] L. Zhang, X. Zou, B. Zhang, L. Cui, J. Zhang, Y. Mao, L. Chen, M. Ji, Theranostics 8, 4129 (2018).

[42] T. Chen, Y. Huang, Small 11, 4998 (2015).

[43] T. Chen, S. Chen, J. Zhou, D. Liang, X. Chen, Y. Huang, Nanoscale 6, 10536 (2014).

[44] T. Chen, F. Lu, A.M. Streets, P. Fei, J. Quan, Y. Huang, Nanoscale 5, 4701 (2013).

[45] J. Li, W. Zhang, T.F. Chung, M.N. Slipchenko, Y.P. Chen, J.X. Cheng, C. Yang, Sci. Rep. 5, 12394 (2015).

[46] P. Samineni, A. deCruz, T.E. Villafańa, W.S. Warren, M.C. Fischer, Opt. Lett. 37, 1310 (2012).

[47] T.E. Villafana, W.P. Brown, J.K. Delaney, M. Palmer, W.S. Warren, M.C. Fischer, Proc. Natl. Acad. Sci. USA 111, 1708 (2014).

[48] T.E. Villafana, J.K. Delaney, W.S. Warren, M.C. Fischer, J. Cult. Herit. 20, 583 (2016).

[49] J. Yu, W.S. Warren, M.C. Fischer, Anal. Chem. 90, 12686 (2018).

[50] J. Yu, W.S. Warren, M.C. Fischer, Sci. Adv. 5, 3136 (2019).

[51] T. Zhu, J.M. Snaider, L. Yuan, L. Huang, Annu. Rev. Phys. Chem. 70, 219 (2019).

[52] E.M. Van Goethem, C.W. Pinion, E.E.M. Cating, J.F. Cahoon, J.M. Papanikolas, ACS Photonics 6, 2213 (2019).

[53] H. Shi, R. Yan, S. Bertolazzi, J. Brivio, B. Gao, A. Kis, D. Jena, H.G. Xing, L. Huang, ACS Nano 7, 1072 (2013).

[54] C.Y. Wong, S.B. Penwell, B.L. Cotts, R. Noriega, H. Wu, N.S. Ginsberg, J. Phys. Chem. C 117, 22111 (2013).
[55] S.S. Lo, T.A. Major, N. Petchsang, L. Huang, M.K. Kuno, G.V. Hartland, ACS Nano 6, 5274 (2012).

[56] E.M. Grumstrup, M.M. Gabriel, E.E.M. Cating, E.M. Van Goethem, J.M. Papanikolas, Chem. Phys. 458, 30 (2015).

[57] B.P. Mehl, J.R. Kirschbrown, R.L. House, J.M. Papanikolas, J. Phys. Chem. Lett. 2, 1777 (2011).

[58] B.P. Mehl, J.R. Kirschbrown, M.M. Gabriel, R.L. House, J.M. Papanikolas, J. Phys. Chem. B 117, 4390 (2013).

[59] E.M. Grumstrup, M.M. Gabriel, C.W. Pinion, J.K. Parker, J.F. Cahoon, J.M. Papanikolas, Nano Lett. 14, 6287 (2014).

[60] B. Gao, G.V. Hartland, L. Huang, ACS Nano 6, 5083 (2012).

[61] Y. Zhu, J.X. Cheng, J. Chem. Phys. 152, 20901 (2020).

[62] M. Zavelani-Rossi, M.G. Lupo, F. Tassone, L. Manna, G. Lanzani, Nano Lett. 10, 3142 (2010).

[63] J.I. Hotta, E. Fron, P. Dedecker et al., J. Am. Chem. Soc. 132, 5021 (2010).

[64] T. Watanabe, Y. Iketaki, M. Sakai, T. Ohmori, T. Ueda, T. Yamanaka, S.I. Ishiuchi, M. Fujii, Chem. Phys. Lett. 420, 410 (2006).

[65] T.A. Klar, S.W. Hell, Opt. Lett. 24, 954 (1999).

[66] E. Rittweger, B.R. Rankin, V. Westphal, S.W. Hell, Chem. Phys. Lett. 442, 483 (2007).

[67] A. Chenu, G.D. Scholes, Annu. Rev. Phys. Chem. 66, 69 (2015).

[68] L. Kastrup, S.W. Hell, Angew. Chem. Int. Ed. 43, 6646 (2004).

[69] N. Accanto, J.B. Nieder, L. Piatkowski, M. Castro-Lopez, F. Pastorelli, D. Brinks, N.F. Van Hulst, Light Sci. Appl. 3, e143 (2014).

[70] S. Berciaud, D. Lasne, G.A. Blab, L. Cognet, B. Lounis, Phys. Rev. B Condens. Matter Mater. Phys. 73, 045424 (2006).

[71] M. Allione, A. Ballester, H. Li, A. Comin, J.L. Movilla, J.I. Climente, L. Manna, I. Moreels, ACS Nano 7, 2443 (2013).

[72] D. Norris, M. Bawendi, Phys. Rev. B Condens. Matter Mater. Phys. 53, 16338 (1996).

[73] M.G. Lupo, F.D. Sala, L. Carbone, M. Zavelani-Rossi, A. Fiore, L. Lüer, D. Polli, R. Cingolani, L. Manna, G. Lanzani, Nano Lett. 8, 4582 (2008). 
[74] G. Xing, Y. Liao, X. Wu, S. Chakrabortty, X. Liu, E.K.L. Yeow, Y. Chan, T.C. Sum, ACS Nano 6, 10835 (2012).

[75] C. Galland, S. Brovelli, W.K. Bae, L.A. Padilha, F. Meinardi, V.I. Klimov, Nano Lett. 13, 321 (2013).

[76] B.T. Diroll, M.E. Turk, N. Gogotsi, C.B. Murray, J.M. Kikkawa, ChemPhysChem 17, 759 (2016).

[77] T. Walz, S.J. Jamieson, C.M. Bowers, P.A. Bullough, C.N. Hunter, J. Mol. Biol. 282, 833 (1998).

[78] S. Brovelli, W.K. Bae, C. Galland, U. Giovanella, F. Meinardi, V.I. Klimov, Nano Lett. 14, 486 (2014).

[79] E. Hendry, M. Koeberg, F. Wang, H. Zhang, C. De Mello Donegá, D. Vanmaekelbergh, M. Bonn, Phys. Rev. Lett. 96, 057408 (2006).

[80] M. Leutenegger, C. Eggeling, S.W. Hell, Opt. Expr. 18, 26417 (2010).

[81] U. Fuchs, U.D. Zeitner, A. Tünnermann, Opt. Expr. 13, 3852 (2005).

[82] R. Trebino, Nat. Photon. 5, 189 (2011).

[83] C. Iaconis, I.A. Walmsley, Opt. Lett. 23, 792 (1998).
[84] N. Accanto, J.B. Nieder, L. Piatkowski, M. Castro-Lopez, F. Pastorelli, D. Brinks, N.F. Van Hulst, Light Sci. Appl. 3, e143 (2014).

[85] N. Accanto, L. Piatkowski, I.M. Hancu, J. Renger, N.F. Van Hulst, Appl. Phys. Lett. 108, 083115 (2016).

[86] D. Brinks, R. Hildner, F.D. Stefani, N.F. van Hulst, Opt. Expr. 19, 25486 (2011).

[87] S. Chong, W. Min, X.S. Xie, J. Phys. Chem. Lett. 1, 3316 (2010).

[88] M. Celebrano, P. Kukura, A. Renn, V. Sandoghdar, Nat. Photon. 5, 95 (2011).

[89] N.P. Mauranyapin, L.S. Madsen, M.A. Taylor, M. Waleed, W.P. Bowen, Nat. Photon. 11, 477 (2017).

[90] P. Kambhampati, J. Phys. Chem. C 115, 22089 (2011).

[91] A. Block, M. Liebel, R. Yu, M. Spector, Y. Sivan, F.J. García De Abajo, N.F. Van Hulst, Sci. Adv. 5, eaav8965 (2019).

[92] S. Deng, D.D. Blach, L. Jin, L. Huang, Adv. Energy Mater. 10 (2020). 